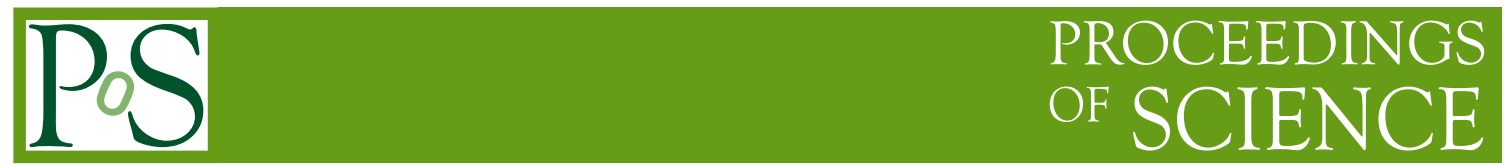

\title{
Boosted top quark measurements at the LHC
}

\author{
Riccardo Di Sipio* \\ University of Toronto \\ E-mail: riccardo.disipio@utoronto.ca
}

In this talk, an overview of measurement performed by the ATLAS and CMS Collaborations at the CERN LHC using high-momentum top quarks is presented. Searches for physics beyond the Standard Model and precision measurements of the Standard Model top-antitop production are discussed.

Sixth Annual Conference on Large Hadron Collider Physics (LHCP2018)

4-9 June 2018

Bologna, Italy

${ }^{*}$ Speaker. 


\section{Introduction}

The large top-quark pair $(t \bar{t})$ production cross-section at the LHC allows detailed studies of the characteristics of top quarks to be performed, providing a unique opportunity to test the Standard Model (SM) at the TeV scale. Furthermore, effects beyond the SM (BSM) can appear as modifications of $t \bar{t}$ total or differential cross-section distributions with respect to the SM predictions, both in cases where top quarks are regarded as signal or as background. As more data is collected, the prospects to measure these distributions for very high-momentum top quarks depends on the ability to reconstruct the highly-boosted decay products of a hadronically-decaying top quark. These techniques, referred to as top-tagging, are at the centre of a very active field of research, playing an increasingly important role in both searches for new physics and in precision measurements of top quarks.

\section{Identification of boosted top quarks}

A number of pattern-recognition algorithms have been devised over the past years to identify ("tag") the hadronic decay of high-momentum top quarks against background processes such as QCD multijet production or the hadronic decay of $W$ and $Z$ bosons $\left(W / Z \rightarrow q \bar{q}^{\prime}\right)$. These algorithms are hence referred to as top-taggers. As the top quark fragments into a three-body decay $(t \rightarrow$ $\left.b W \rightarrow b q \bar{q}^{\prime}\right)$, top-tagging algorithms search for a three-prong structure in the distribution of the final state reconstructed objects associated with a candidate jet, which is in turn reconstructed with large distance parameter (often referred to as large- $R$ jet). The typical pattern in the fully-hadronic final state is determined by the energy and angular distribution of the three quarks originated from the decay of the top quark, which is known or at least calculable based on the knowledge of the Standard Model interactions. This pattern can be statistically distinguished from other processes using a number of techniques such as trimming [1] (which removes pileup by discarding narrow embedded subjets with $p_{T}<5 \%$ of the large- $R$ jet) and a subsequent set of selection cuts based on the jet kinematics [2] (usually transverse momentum, rapidity and mass) and on the value of some substructure variable [3] (such as $N$-subjettiness ratio $\tau_{32}$ or splitting scale $\sqrt{d_{12}}$ ).

\section{Searches for phenomena beyond the Standard Model}

The most straightforward way to look for physics beyond the Standard Model is to search for particular structures in the invariant mass distribution of top-quark pairs, typically in the range of a few $\mathrm{TeV}$, which would indicate the presence of a resonance of a certain mass. The Standard Model predicts a smooth, steeply falling distribution. On the other hand, the presence of additional particles can appear either as a narrow peak, as in the case of a leptophobic $Z^{\prime}$, or a broad peak visible only as a generalized enhancement of the cross-section, due for example by the production of Kaluza-Klein gluons $\left(g_{\mathrm{KK}}\right)$. No excess over the estimated SM background was found in measurements done by the ATLAS [2] and CMS Collaborations [4], as shown in Fig.1.

Similar resonance structures may appear in the invariant mass spectrum of a top quark in association with a $b$ quark, a vector boson $(W, Z)$ or a Higgs boson $(H)$. For example, vectorlike quarks (VLQ), hypothetical spin-1/2 particles that transform as triplets under the color gauge 


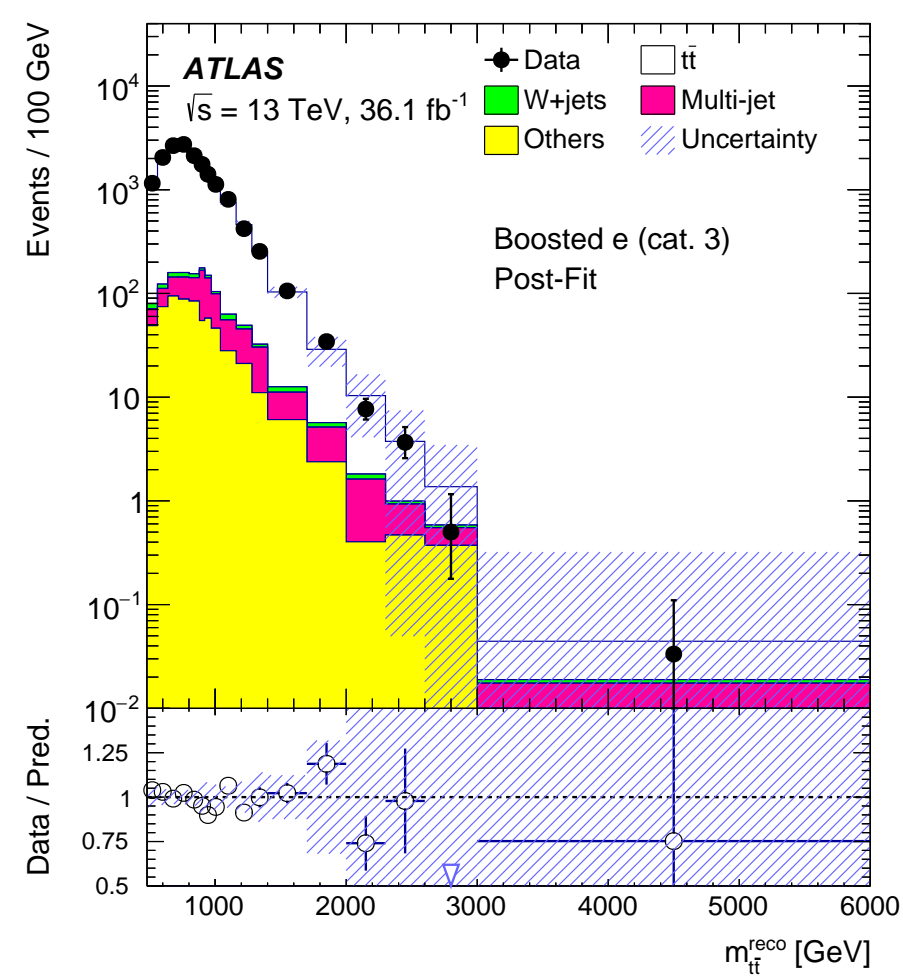

Figure 1: Top-quark pair invariant mass spectrum in the lepton+jets boosted channel at $\sqrt{s}=13 \mathrm{TeV}$, after a likelihood fit under the background-only hypothesis.

group, can be produced either in pairs or singly, and can decay into these final states. Fig. 2 shows for example the invariant mass of a di-jet system where one jet is top-tagged and the other is Higgstagged, representing the decay $T \rightarrow t H$. Another possibility is given by additional gauge bosons $\left(W^{\prime}, Z^{\prime}\right)$, mediators of new charged vector currents, which may decay into a $t b$ pair. No excess over the estimated SM background was found in measurements done by the ATLAS [5, 6] and CMS Collaborations [7].

An even more exotic search is inspired by Supersymmetric extensions of the Standard Model (SUSY), where the hypothetical scalar partner of the top quark $(\tilde{t})$ decays into a neutralino $\left(\tilde{\chi}^{0}\right)$ and a Standard Model top quark. Since the neutralino does not interact with the detector, the experimental signature of the pair production is given by two top quarks and large missing transverse energy $\left(\mathbb{E}_{T}\right)$. The signal cross-section depends on the $\tilde{t}$ and $\tilde{\chi}^{0}$ masses in a characteristic way which allows to set limits on certain (simplified) SUSY models [8].

Finally, it is possible to construct models that attempt to explain the origin of Dark Matter in terms of additional scalar and vector fields, which would appear at the LHC as final states with just an isolated top quark in association with large $\mathbb{E}_{T}$. No evidence for this process has been found by the CMS Collaboration and limits on the mass of dark matter candidates have been set based on the $\mathbb{E}_{T}$ spectrum [9]. 


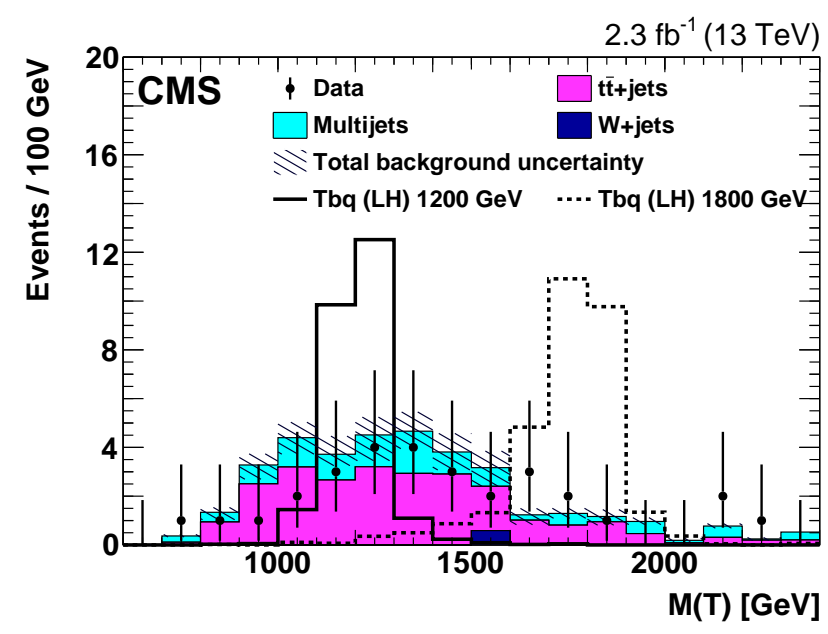

Figure 2: Invariant mass distribution of the $(t, H)$ pair at $\sqrt{s}=13 \mathrm{TeV}$. The simulated $T$ quark signal distributions for two $T$ quark masses are also shown, for which the values of the product of the signal cross sections and the branching fraction $\mathrm{B}(T \rightarrow t H)$ are taken to be $1 \mathrm{pb}$.

\section{Precision Standard Model measurements}

In the Standard Model, top-quark pairs are mostly produced at low rapidity ("central" production) in a back-to-back configuration. The cross-section decreases exponentially as a function of the transverse momentum. Any deviation from this behavior would signal either an incomplete understanding of the Standard Model process, or the presence of additional particles not included in the Standard Model itself. Assuming only Standard Model production, state-of-the art comparisons against theoretical models are performed in a fiducial phase-space (particle level), typically to test next-to-leading order (NLO) Monte Carlo event generators and tune their parameters, or in the full phase-space (parton level), to compare data against next-to-next-leading order (NNLO) calculations, for which matching to parton shower algorithms is not yet available for top quark production.

The most important observable is the transverse momentum, which spans different kinematic regimes and reconstruction techniques from 0 up to about $1 \mathrm{TeV}$. Sensitive to final state radiation, very precise low- $p_{\mathrm{T}}$ differential cross-sections indicate a disagreement with NLO calculations which becomes more and more evident with increasing $p_{\mathrm{T}}$, a situation that is worth investigating in the high-momentum regime. Both ATLAS [10] and CMS [11] performed such measurements looking for high-momentum top quarks in data taken at $\sqrt{s}=8 \mathrm{TeV}$. In this case, predictions obtained using Monte Carlo event generators (MadGraph [12], POWHEG [13] and MC@NLO [14], all interfaced to PYTHIA 6 [15]) tend to be in agreement with the data. However, as can be seen in Fig.3, the large systematic uncertainties (large- $R$ jet energy and mass scale, top-tagging and signal modeling) do not allow yet a test as stringent as in the low- $p_{\mathrm{T}}$ case.

The differential cross-sections as a function of $t \bar{t}$ system variables such as the invariant mass or the transverse momentum are critical to reduce the uncertainties of top quark production in the context of searches for beyond the Standard Model physics. The ATLAS Collaboration mea- 
sured several $t \bar{t}$ differential cross-section distributions at $\sqrt{s}=13 \mathrm{TeV}$ in the all-hadronic final state [16]. A general good agreement was found for most of the generators (POWHEG-BOX [17] interfaced to either Pythia8 [18] or Herwig7 [19], Sherpa [20]), with the exception of MadGraph5_aMC@NLO [21] interfaced to Pythia8 in observables related to the emission of very energetic radiation as shown in Fig.4.

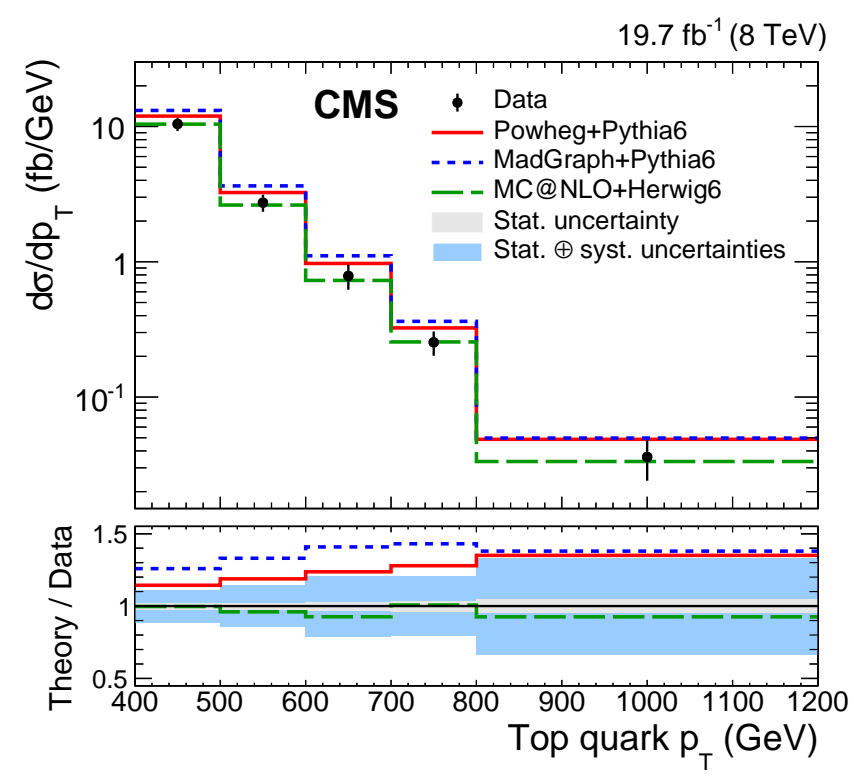

Figure 3: Full phase-space top-quark pairs differential cross-section as a function of the top-quark transverse momentum $\left(p_{\mathrm{T}}\right)$ in the lepton+jets boosted channel at $\sqrt{s}=8 \mathrm{TeV}$.

\section{Conclusions}

ATLAS and CMS collected data containing a large number of high-momentum top-quark pairs, which are now routinely deployed for searches and precision measurements. Boosted top quarks are excellent probes for high-mass regions, where new physics is more likely to appear. Precision measurements support searches and help improving our understanding of the $t \bar{t}$ production.

\section{References}

[1] Jet trimming, D. Krohn, J. Thaler, L.-T. Wang, JHEP 02 (2010) 084

[2] Search for heavy particles decaying into top-quark pairs using lepton-plus-jets events in proton-proton collisions at $\sqrt{s}=13 \mathrm{TeV}$ with the ATLAS detector, The ATLAS Collaboration, Eur. Phys. J. C (2018) 78

[3] Measurement of jet substructure observables in $t \bar{t}$ events from $p p$ collisions at $\sqrt{s}=13 \mathrm{TeV}$, The CMS Collaboration, CMS-PAS-TOP-17-013

[4] Search for $t \bar{t}$ resonances in highly boosted lepton+jets and fully hadronic final states in proton-proton collisions at $\sqrt{s}=13 \mathrm{TeV}$, The CMS Collaboration, JHEP 07 (2017) 001 


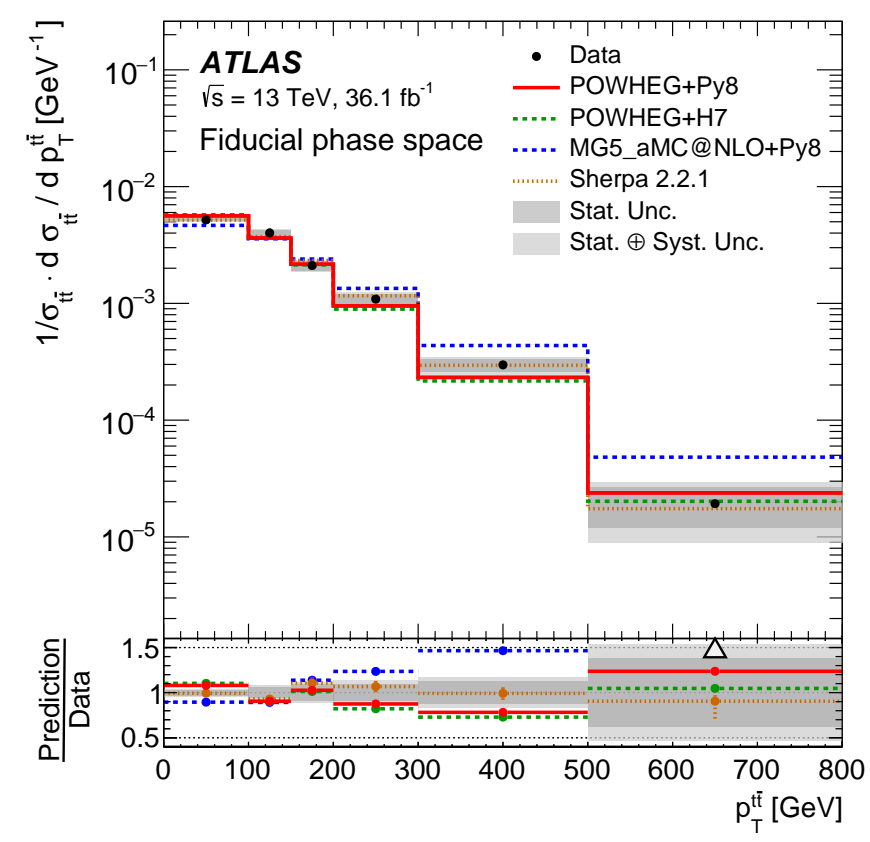

Figure 4: Fiducial phase-space top-quark pairs normalized differential cross-section as a function of the $t \bar{t}$ system transverse momentum in the all-hadronic boosted channel at $\sqrt{s}=13 \mathrm{TeV}$.

[5] Search for pair production of up-type vector-like quarks and for four-top-quark events in final states with multiple b-jets with the ATLAS detector, The ATLAS Collaboration, JHEP 07 (2018) 089

[6] Search for $W^{\prime} \rightarrow t b$ decays in the hadronic final state using $p p$ collisions at $\sqrt{s}=13 \mathrm{TeV}$, The ATLAS Collaboration, Phys. Lett. B 781 (2018) 327

[7] Search for electroweak production of a vector-like quark decaying to a top quark and a Higgs boson using boosted topologies in fully hadronic final states, The CMS Collaboration, JHEP 04 (2017) 136

[8] Search for a scalar partner of the top quark in the jets plus missing transverse momentum final state at $\sqrt{s}=13 \mathrm{TeV}$ with the ATLAS detector, The ATLAS Collaboration, JHEP 12 (2017) 085

[9] Search for dark matter in events with energetic, hadronically decaying top quarks and missing transverse momentum at $\sqrt{s}=13 \mathrm{TeV}$, The CMS Collaboration, JHEP 06 (2018) 027

[10] Measurement of the differential cross-section of highly boosted top quarks as a function of their transverse momentum in $\sqrt{s}=8 \mathrm{TeV}$ proton-proton collisions using the ATLAS detector, The ATLAS Collaboration, Phys. Rev. D 93, 032009 (2016)

[11] Measurement of the integrated and differential $t \bar{t}$ production cross sections for high- $p_{\mathrm{T}}$ top quarks in pp collisions at $\sqrt{s}=8 \mathrm{TeV}$, The CMS Collaboration, Phys. Rev. D 94, 072002 (2016)

[12] MadGraph 5 : Going Beyond, J. Allwall et al., JHEP 06 (2011) 128

[13] Matching NLO QCD computations with Parton Shower simulations: the POWHEG method, S. Frixione, P. Nason and C. Oleari, JHEP 11 (2007) 070

[14] Matching NLO QCD computations and parton shower simulations, S. Frixione, B.R. Webber, JHEP 06 (2002) 029 
[15] PYTHIA 6.4 Physics and Manual, T. Sjöstrand, S. Mrenna and P. Z. Skands, JHEP 05 (2006) 026

[16] Measurements of $t \bar{t}$ differential cross-sections of highly boosted top quarks decaying to all-hadronic final states in $p p$ collisions at $\sqrt{s}=13 \mathrm{TeV}, \mathrm{TeV}$ using the ATLAS detector, The ATLAS Collaboration, Phys. Rev. D 98, 012003 (2018)

[17] A general framework for implementing NLO calculations in shower Monte Carlo programs: the POWHEG BOX, S. Alioli, P. Nason, C. Olearic, E. Re, JHEP 06 (2010) 043

[18] A Brief Introduction to PYTHIA 8.1, T. Sjöstrand, S. Mrenna and P. Z. Skands, Comput. Phys. Commun. 178 (2008) 852

[19] Herwig 7.0/Herwig++ 3.0 release note, J. Bellm et al., Eur. Phys. J. C 76 (2016) 196

[20] Event generation with SHERPA 1.1, T. Gleisberg et al., JHEP 02 (2009) 007

[21] The automated computation of tree-level and next-to-leading order differential cross sections, and their matching to parton shower simulations, J. Allwall et al., JHEP 07 (2014) 079 